\title{
Morphological and Structural Study of GaAs Nanowires Grown Using VLS Method on EBL Patterned Au Catalysts
}

B. O’Dowd ${ }^{1,2}$, T. Wojtowicz ${ }^{3}$, S. Rouvimov ${ }^{4}$, X. Liu ${ }^{1}$, R. Pimpinella ${ }^{1}$, V. Kolkovsky ${ }^{3}$, T. Wojciechowski $^{3}$, M. Zgirski ${ }^{3}$, M. Dobrowolska ${ }^{1}$ and J. Furdyna ${ }^{1}$

1. Physics, University of Notre Dame, IN, USA

2. School of Physics, Trinity College Dublin, Ireland

3. Institute of Physics, Polish Academy of Sciences, Warsaw, Poland

4. Notre Dame Integrated Imaging Facility (NDIIF), University of Notre Dame, IN, USA

Semiconductor nanowires have attracted considerable interest due to their large range of potential applications and their ability to exhibit interesting fundamental physical properties which may be imperceptible in the bulk. The vapour-liquid-solid (VLS) mechanism is perhaps the most promising fabrication mechanism for fabrication of such nanowires in terms of reproducibility and quality of structures grown[1]. Despite a great deal of research in this area, however, there remain central unanswered questions about the specific processes that govern this growth mode. Here we present a study of the growth of GaAs nanowires grown using the VLS process on (111)B GaAs substrates as a function of source pressure and substrate temperature. The morphology and crystal structure are confirmed using scanning electron microscopy (SEM), scanning transmission microscopy (STEM) and high-resolution transmission electron microscopy (HRTEM).

We grew GaAs nanowires (NWs) in a Riber $32 \mathrm{MBE}$ system on GaAs (111)B substrates with $100 \times$ $100 \mu \mathrm{m}$ grids of EBL-defined Au nanodots. There are 12 grids on each sample with dot diameters of 30 , 70 and $110 \mathrm{~nm}$ and dot-to-dot separations of $0.5,1.0,1.5$ and $2 \mu \mathrm{m}$. Growth is also performed simultaneously on a similar substrate with a $1 \mathrm{~nm}$ layer of Au covering the entire surface. Upon heating, this layer forms a disordered array of nanodots, which produce wires used for TEM analysis and for comparing the wires grown by this method with those obtained on the patterned sample. Four samples (A,B, C and D) were prepared by this procedure. A and B were grown with a substrate temperature of $470^{\circ} \mathrm{C}$, while $\mathrm{C}$ and $\mathrm{D}$ were grown at $520^{\circ} \mathrm{C}$. Ga flux was kept constant for all four samples, while As flux was higher for samples $\mathrm{B}$ and $\mathrm{D}$ than for samples $\mathrm{A}$ and $\mathrm{C}$.

SEM images of a wire from each of the four samples taken at $45^{\circ}$ are shown in Figure $1 \mathrm{~A}(\mathrm{i}), 1 \mathrm{~B}(\mathrm{i})$, $1 C(i)$ and $1 D(i)$. Wire A has a tapered base, followed by a more uniform 'neck' before reaching the AuGa droplet. Wire B has a uniform tapering, resulting in a needle-like shape. $\mathrm{C}$ has a slight tapering which depends on the length of the wire, and unusually is wider at the top for tall $(>3 \mu \mathrm{m})$ wires. Wire $\mathrm{D}$ has a uniform hexagonal structure followed by a sharp tip. Of interest to us from the point of view of the growth process and adatom diffusion are the facets that make up the sidewalls of the NWs, since adatoms have different diffusion barriers on different crystal surfaces[2]. By correlating SEM and TEM for each sample, we can confirm that the structure of the wires is wurtzite for all four samples (with some zinc-blende defects), and that those wires grown at low temperature (A and B) have $\{10 \overline{1} 0\}$ facets, while those grown at high temperature $(C$ and $D)$ have $\{11 \overline{2} 0\}$ facets. 
HRTEM images are shown in Figure 1A(ii),(iii), 1B(ii), 1C(ii),(iii) and 1D(ii). From these we can see that the wire structure is generally wurtzite, as expected theoretically for narrow NWs[3]. In the tapered base of wire A we see a high density of stacking defects, and in wire B we see that the gradual tapering is accommodated by narrow $(\approx 1 \mathrm{~nm})$ zinc-blende $(\mathrm{ZB})$ defects. Wire $\mathrm{C}$ has a high density of defects, switching regularly between $\mathrm{WZ}$ and $\mathrm{ZB}$, while wire $\mathrm{D}$ is uniformly $\mathrm{WZ}$ with an extremely low density of defects, even at the transition to the sharp tip region of the wire, which had previously been reported to have a ZB structure[3]. These results, particularly for wire D, show an exceptional quality of structure, with exciting possibilities for nano-electronic applications.

Energy-dispersive X-ray spectroscopy (EDX) is used to investigate the chemical composition of the droplet at the top of the wire. For wire B we see that the Au-Ga ratio is approximately 3:1, as expected for a eutectic alloy of these elements, while for wire $\mathrm{C}$ the droplet is mostly excess $\mathrm{Ga}$, with a small AuGa droplet inside (see Figure 1C(ii)).

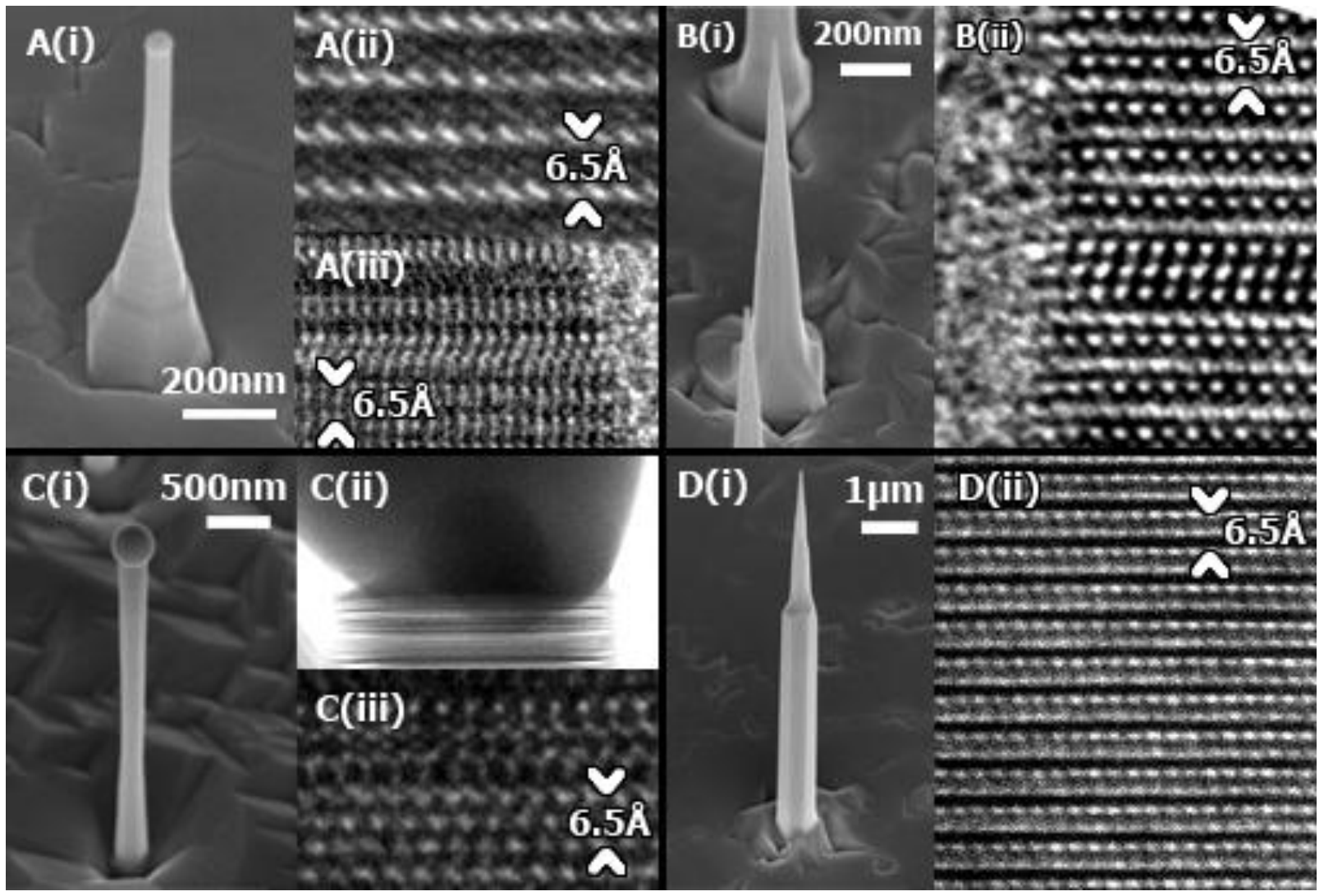

Figure 1. SEM and HRTEM images of NWs from the four samples taken at $45^{\circ}$ to the surface. A highly reproducible, yet diverse, range of morphologies are possible. Growth conditions for the four samples are described in the text.

[1] K. Tivakornsasithorn et al, Journal of Vacuum Science \& Technology B: Microelectronics and Nanometer Structures, 30, no. 2, 2012, p. 02B115.

[2] V. Pankoke, S. Sakong, and P. Kratzer, Physical Review B, 86, no. 8, Aug. 2012, p. 085425.

[3] F. Glas, J.-C. Harmand, and G. Patriarche, Physical Review Letters, 99, no. 14, Oct. 2007, p. 3 